06

\title{
Технология роста и характеристики полученных тонких пленок иридата стронция и гетероструктур иридат-купратный сверхпроводник
}

\author{
(C) A.M. Петржик ${ }^{1}$, G. Cristiani ${ }^{2}$, Г. Логвенов ${ }^{2}$, А.E. Пестун ${ }^{3}$, \\ Н.В. Андреев ${ }^{3}$, Ю.В. Кислинский ${ }^{1}$, Г.А. Овсянников ${ }^{1}$ \\ ${ }^{1}$ Институт радиотехники и электроники им. В.А. Котельникова РАН, \\ Москва, Россия \\ ${ }^{2}$ Max Planck Institute for Solid State Research, 70569, Stuttgart, Germany \\ ${ }^{3}$ Национальный исследовательский технологический университет \\ „МИСиС“, Москва, Россия \\ E-mail: petrzhik@hitech.cplire.ru \\ Поступило в Редакцию 10 января 2017 г.
}

Предложена и отработана технология эпитаксиального роста тонких пленок иридата стронция $\mathrm{Sr}_{2} \mathrm{IrO}_{4}$ и гетероструктур $\mathrm{Sr}_{2} \mathrm{IrO}_{4} / \mathrm{YBa}_{2} \mathrm{Cu}_{3} \mathrm{O}_{7-\delta}$, содержащих купратный сверхпроводник. Показано, что рост двухслойной структуры происходит эпитаксиально, а слой купратного сверхпроводника имеет ту же критическую температуру, что и автономная пленка $(\sim 91 \mathrm{~K})$. Кристаллографические параметры полученных пленок иридата близки к табличным значениям, температурные зависимости сопротивления тонких пленок согласуются с литературными данными.

DOI: 10.21883/PJTF.2017.12.44705.16704

Иридат стронция $\mathrm{Sr}_{2} \mathrm{IrO}_{4}$ обладает рядом интересных свойств, большинство из которых связывают с характерным для этого материала сильным спин-орбитальным взаимодействием [1]. К таким свойствам главным образом следует отнести недавно обнаруженную $d$-волновую щель в электронной плотности состояний [2], уникально высокое значение анизотропного магнетосопротивления [3] и возможность образования состояния топологического изолятора [4]. Несмотря на довольно активное изучение данного материала в последнее время, еще не все теоретически предсказанные эффекты были подтверждены экспериментально. В частности, экспериментально не был подтвержден 
эффект возникновения спин-триплетного спаривания при пропускании электрического тока через структуру, содержащую прослойку из $\mathrm{Sr}_{2} \mathrm{IrO}_{4}$ и два сверхпроводящих электрода. Также пока только теоретически была рассмотрена реализация синглет-триплетного преобразователя в структуре $S-\mathrm{Sr}_{2} \mathrm{IrO}_{4}-S^{\prime}$, где $S-$ синглетный, а $S^{\prime}$ - триплетный сверхпроводник [5].

Монокристаллы $\mathrm{Sr}_{2} \mathrm{IrO}_{4}$ показывают диэлектрический тип зависимости сопротивления от температуры и переходят в нескомпенсированное антиферромагнитное состояние при температурах ниже $240 \mathrm{~K}$ [6]. Магнитные свойства тонких пленок несколько отличаются от монокристаллов и сильно зависят от используемых методов синтеза и механических напряжений, вызванных рассогласованием кристаллографических параметров подложки и пленки [7]. Кристаллографические и транспортные свойства также имеют некоторые особенности [8-10]. Кристаллическая структура монокристаллов слоистая, типа $\mathrm{La}_{2} \mathrm{CuO}_{4}$, имеет тетрагональную симметрию с пространственной группой $I 4 / \mathrm{mmm}$, в отсутствие напряжений параметры решетки $a=3.888 \AA, c=12.90 \AA$ [8]. Фаза $\mathrm{Sr}_{2} \mathrm{IrO}_{4}$ является метастабильной и в отличие от стабильной немагнитной $\mathrm{SrIrO}_{3}$ и купратных ВТСП требует нестандартного для оксидов режима синтеза, а именно очень низкого давления кислорода и высокой температуры напыления [8-10].

Целью настоящей работы являлись разработка методики эпитаксиального роста пленок $\mathrm{Sr}_{2} \mathrm{IrO}_{4}$ и двухслойных гетероструктур $\mathrm{YBa}_{2} \mathrm{Cu}_{3} \mathrm{O}_{7-\delta}$ (далее $\left.\mathrm{YBCO}\right) / \mathrm{Sr}_{2} \mathrm{IrO}_{4}$ и исследование физических свойств полученных образцов для дальнейшего экспериментального изучения эффекта синглет-триплетного преобразования, предсказанного в работе [5], а также других возможных особенностей.

Следует отметить, что выбор ҮВСО в качестве одного из сверхпроводящих электродов не случаен: в структуре $\mathrm{Nb} / \mathrm{Au} /$ $\mathrm{La}_{0.7} \mathrm{Sr}_{0.3} \mathrm{MnO}_{3} / \mathrm{SrRuO}_{3} / \mathrm{YBCO}$ уже наблюдался сверхпроводящий ток, который был объяснен проникновением дальнодействующей триплетной компоненты сверхпроводящего параметра порядка в магнитную прослойку [11]. Исходя из результатов, полученных в работе [11], не исключено, что для обнаружения спин-триплетного сверхпроводящего тока может оказаться достаточным изготовление структуры, состоящей из исключительно синглетных сверхпроводников, например структуры $\mathrm{Nb} / \mathrm{Au} / \mathrm{Sr}_{2} \mathrm{IrO}_{4} / \mathrm{YBCO}$. Для создания такой структуры наибольшую тех-

Письма в ЖТФ, 2017, том 43, вып. 12 


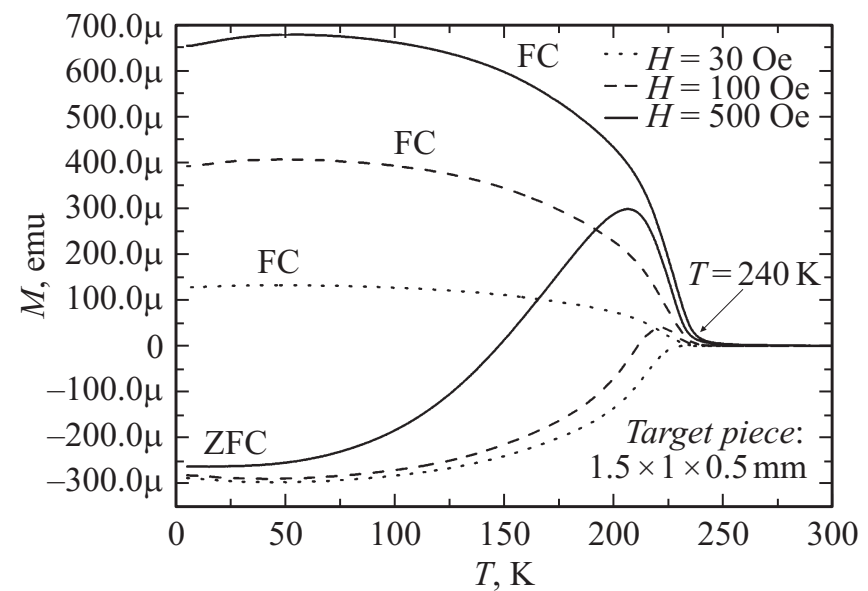

Рис. 1. Зависимость величины намагниченности $M$ мишени $\mathrm{Sr}_{2} \mathrm{IrO}_{4}$ размером $1.5 \times 1 \times 0.5 \mathrm{~mm}$ от температуры при следующих режимах измерений: $\mathrm{FC}-$ заморозка в магнитном поле, ZFC - заморозка в нулевом магнитном поле. Температура перехода в антиферромагнитное состояние $240 \mathrm{~K}$.

нологическую сложность представляет качественный рост двухслойной гетероструктуры $\mathrm{Sr}_{2} \mathrm{IrO}_{4} / \mathrm{YBCO}$.

Тонкие пленки $\mathrm{Sr}_{2} \mathrm{IrO}_{4}$ наносились на различные подложки методом лазерной абляции мишени. Мишени изготавливались из смеси порошков $\mathrm{SrCO}_{3}$ и $\mathrm{IrO}_{2}$ по стандартной керамической технологии. Исходный состав смеси до проведения синтеза $\mathrm{Sr}_{1.85} \mathrm{IrO}_{4}$ После перемешивания полученный порошок прессовался в таблетки $\left(P_{\text {press }}=1 \mathrm{t} / \mathrm{cm}^{2}\right)$, затем проводилась термообработка при $900^{\circ} \mathrm{C}(22 \mathrm{~h})$ и при $1000^{\circ} \mathrm{C}(22 \mathrm{~h})$ с промежуточным измельчением и повторным прессованием. После завершения синтеза для приготовления мишеней полученный порошок смешивался со связкой (парафин, растворенный в гексане, массовая доля гексана 5\%) и высушивался при температуре $120^{\circ} \mathrm{C}$ в течение $2 \mathrm{~h}$. После сушки смесь повторно измельчали и прессовали в таблетки диаметром $12 \mathrm{~mm}$ и толщиной $3.6 \mathrm{~mm}\left(P_{\text {press }}=2 \mathrm{t} / \mathrm{cm}^{2}\right)$. Далее таблетки спекались при $1180^{\circ} \mathrm{C}$ в течение $20 \mathrm{~h}$. Рентгенофазовый анализ показал, что полученные мишени являются однофазными и имеют тетрагональную кристаллическую решетку: пространственная

Письма в ЖТФ, 2017, том 43, вып. 12 
группа I4/mmm, параметры решетки: $a=3.884 \AA, c=12.891 \AA$. Полученные параметры близки к табличным значениям для монокристаллов, которые составляют соответственно 3.888 и $12.90 \AA$ согласно работе [8], в работе [12] представлены несколько иные параметры: $a=3.885 \AA, c=12.918 \AA$. СКВИД-магнетометр (рис. 1) показывает переход мишени в антиферромагнитное состояние при $240^{\circ} \mathrm{K}$, что соответствует литературным данным. Пересчитанная из етu величина намагниченности составляет $0.014 \mu \mathrm{B} / \mathrm{Ir}$. Наиболее близкое значение $M=0.023 \mu \mathrm{B} / \mathrm{Ir}$ дается в обзоре [13], нейтронные исследования [6] дают на порядок более высокие значения. Таким образом, учитывая близкие к табличным параметры кристаллической решетки и температуры перехода в антиферромагнитное состояние (температуры Нееля) синтезированной мишени, можно считать, что изготовленная мишень действительно имела необходимый для роста пленок $\mathrm{Sr}_{2} \mathrm{IrO}_{4}$ состав.

Напыление пленок иридата стронция производилось с помощью $\mathrm{KrF}$ эксимерного лазера. В работах [8-10] для синтеза также использовался эксимерный лазер с длиной волны $248 \mathrm{~nm}$, напыление производилось в атмосфере кислорода при очень низком давлении 0.0013-0.05 mbar и температурах подложки от 700 до $850^{\circ} \mathrm{C}$. Первая пленка $\mathrm{Sr}_{2} \mathrm{IrO}_{4}$ была выращена в подобном режиме при давлении кислорода $0.05 \mathrm{mbar}$ на подложке (100) $\mathrm{SrTiO}_{3}$. Температура подложки контролировалась пирометром, частота импульсов составляла $2 \mathrm{~Hz}$, энергия $-1.6 \mathrm{~J} / \mathrm{cm}^{2}$, количество импульсов - 2000. После напыления образец выдерживался при $500^{\circ} \mathrm{C}$ в атмосфере кислорода в течение $30 \mathrm{~min}$. Из рентгеновской дифрактограммы на рис. 2, $a$ видно, что дифракционный пик от пленки сильно размыт и наряду с основной фазой $\mathrm{Sr}_{2} \mathrm{IrO}_{4}$ обнаруживается побочная фаза, вероятно, состава $\mathrm{SrIrO}_{3}$. Чтобы не допустить выпадение побочной фазы, требовалось снизить давление кислорода и, возможно, увеличить нагрев подложки. Но поддерживать стабильное давление кислорода в районе $0.01 \mathrm{mbar}$ и ниже являлось сложной с технической точки зрения задачей, поэтому было решено заменить кислородную атмосферу на аргонную. Было показано (рис. 2, a), что в атмосфере аргона пик от побочной фазы не наблюдается, а $c$-параметр наблюдаемой фазы равен $12.83 \AA$ (толщина пленки $17 \mathrm{~nm}$ ), что даже лучше соответствует табличным для монокристалла $\mathrm{Sr}_{2} \mathrm{IrO}_{4}$ значениям $c$-параметра $(12.90 \AA)$, чем полученные в [8] $12.75 \AA$ (толщина пленки $18 \mathrm{~nm}$ ) для той же подложки $\mathrm{SrTiO}_{3}$. В дальнейшем температура подложки была еще несколько увеличена. Наиболее совершенная 

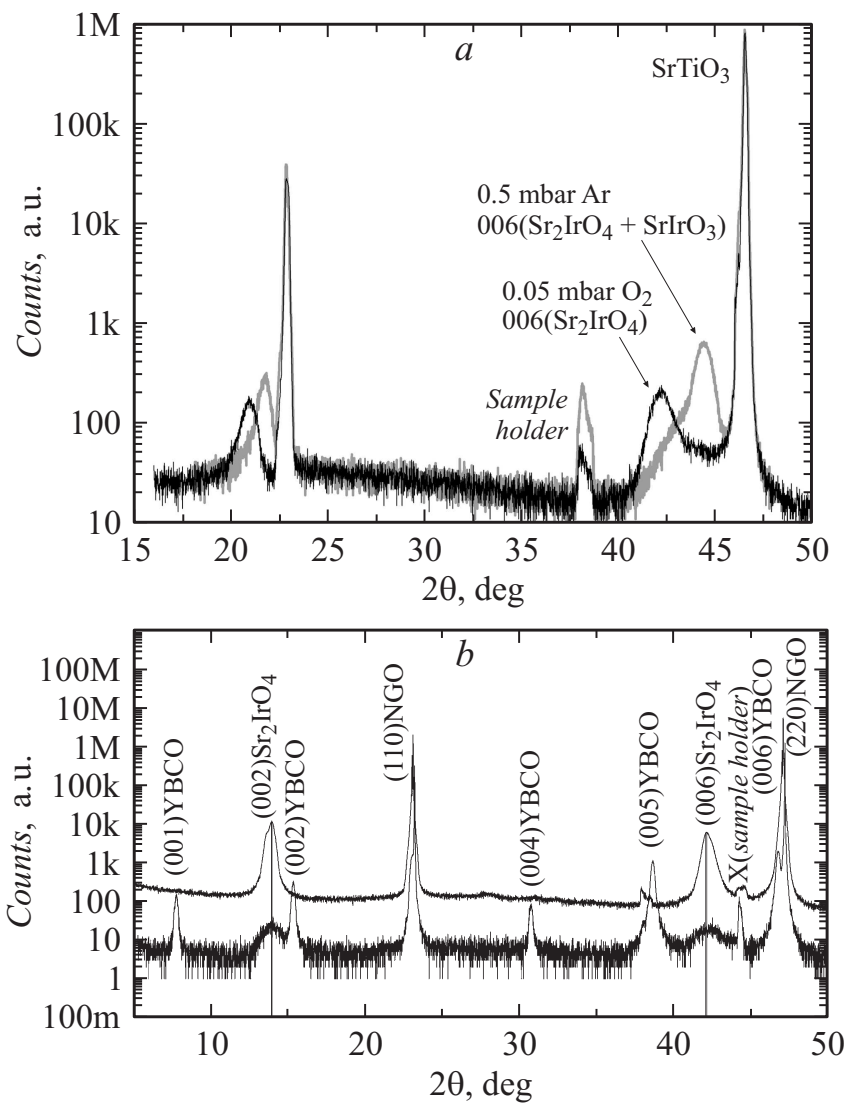

Рис. 2. $a-$ рентгеновская дифрактограмма автономных пленок $\mathrm{Sr}_{2} \mathrm{IrO}_{4}$ толщиной $17 \mathrm{~nm}$, нанесенных методом лазерной абляции на подложку (100) $\mathrm{SrTiO}_{3}$ при разных условиях: с использованием атмосферы кислорода ( $0.05 \mathrm{mbar})$ и атмосферы аргона $(0.5 \mathrm{mbar})$, дифрактограммы сняты в режиме брэгговского отражения; $b-$ рентгеновские дифрактограммы, снятые в режиме брэгговского отражения, для автономной пленки $\mathrm{Sr}_{2} \mathrm{IrO}_{4} /(110) \mathrm{NdGaO}_{3}$ (верхний спектр) и гетероструктуры $\mathrm{Au} / \mathrm{Sr}_{2} \mathrm{IrO}_{4} / \mathrm{YBCO} /(110) \mathrm{NdGaO}_{3}$ (нижний спектр).

структура пленок вырастала при $T=800-760^{\circ} \mathrm{C}$. В этом режиме пленки наносились на 4 типа подложек: (100) $\mathrm{SrTiO}_{3},(110) \mathrm{NdGaO}_{3}$,

Письма в ЖТФ, 2017, том 43, вып. 12 
(100) $\mathrm{La}_{0.3} \mathrm{Sr}_{0.7} \mathrm{Al}_{0.65} \mathrm{Ta}_{0.35} \mathrm{O}_{3}$ (LSAT) и (100) $\mathrm{LaAlO}_{3}$. При равной толщине $34 \mathrm{~nm}$ наиболее близкую к объемному монокристаллу структуру имели пленки на подложках (100) $\mathrm{SrTiO}_{3}$ и (110) $\mathrm{NdGaO}_{3}, c$-параметр таких пленок - $12.87 \AA$ (рис. $2, b$, верхний спектр). Таким образом, было показано, что синтез пленок $\mathrm{Sr}_{2} \mathrm{IrO}_{4}$ в атмосфере аргона возможен и, вероятно, даже более прост с технологической точки зрения.

С помощью электрометра Keithley 6517B и температурного контроллера Neocera LTC-21 были измерены зависимости удельного coпротивления от температуры для пленок иридата стронция, выращенных на подложках LSAT и $\mathrm{SrTiO}_{3}$. Эти подложки обеспечивают наименьшее рассогласование кристаллографических параметров [9]. Измерялись пленки толщиной 17 и $34 \mathrm{~nm}$, нанесенные на LSAT, и пленка толщиной $34 \mathrm{~nm}$, нанесенная на $\mathrm{SrTiO}_{3}$. Удельные сопротивления пленок при комнатной температуре на несколько порядков превышают значения, представленные в работе [8], что может быть связано с различием в режимах нанесения пленок. На рис. 3, a представлена зависимость удельного сопротивления от обратной температуры. Линейные участки кривых соответствуют механизму активационной (диэлектрической) проводимости, для которой характерна зависимость $\rho=\rho_{0} \exp \left(\Delta E_{g} / 2 k T\right)$ [8]. Здесь $\Delta E_{g}$ - величина диэлектрической щели, которая для наших пленок составляла $208-230 \mathrm{meV}$, а $k-$ константа Больцмана. Из рисунка видно, что описанный механизм характерен для низких температур. Для диапазона очень низких температур (выше пунктирной линии на рис. $3, a$ ) наблюдаются утечки в измерительном стенде, вклад которых становится заметен при $R>50 G \Omega$. При повышении температуры в дополнение к активационному подключается еще один механизм проводимости. Предположительно этот механизм является 3-мерной проводимостью с переменной длиной прыжка [8], для которого характерна зависимость $\rho=\rho_{0} \exp \left[\left(T_{0} / T\right)^{1 / 4}\right]$. Температурные участки, на которых данный механизм становится преобладающим, линейны в координатах $\rho$ от $T^{-1 / 4}$ и хорошо видны на рис. $3, b$. 3-мерная прыжковая проводимость в тонких пленках может возникать только при условии $r<d$, где $r$ - длина прыжка, а $d-$ толщина пленки. Длина прыжка падает с ростом температуры, т. е. 3-мерная проводимость может существовать лишь выше некоторой температуры $T_{\mathrm{VRH}}$, ниже которой прыжки становятся двухмерными. Длина прыжка при минимальной температуре участка предположительно прыжковой проводимости была оценена по формуле $r=a\left(T_{0} / T\right)^{1 / 4}$, где $a$ - радиус локализации. Длина 
прыжка составляет $13-17 \mathrm{~nm}$ и не превышает толщин рассматриваемых пленок, что подтверждает возможность возникновения механизма 3-мерной прыжковой проводимости. Для образца толщиной $17 \mathrm{~nm}$ (LSAT) $T_{\mathrm{VRH}} \approx 250 \mathrm{~K}$, константа $T_{0}=351 \cdot 10^{6} \mathrm{~K}$ (рис. $3, b$ ), отсюда радиус локализации $a=d\left(T_{\mathrm{VRH}} / T_{0}\right)^{1 / 4}=0.5 \mathrm{~nm}$. Близкие параметры трехмерной прыжковой проводимости были получены в работе [14] для манганитов $\mathrm{La}(\mathrm{Ba}) \mathrm{MnO}_{3}$.

Экспериментально были подобраны оптимальные режимы роста 2-слойной структуры $\mathrm{Sr}_{2} \mathrm{IrO}_{4} / \mathrm{YBCO}$, с одной стороны, сохраняющие сверхпроводящие свойства слоя ҮВСО, а с другой - обеспечивающие рост метастабильной фазы иридата $\mathrm{Sr}_{2} \mathrm{IrO}_{4}$. Кристаллографические параметры контролировались методом рентгеновской дифракции, а сверхпроводящие свойства - измерением температуры перехода в сверхпроводящее состояние системой на основе принципа взаимной индукции двух магнитных катушек. Подложка (100) $\mathrm{SrTiO}_{3}$ была заменена на более перспективную для создания меза-структур (110) $\mathrm{NdGaO}_{3}$, так как известно, что (100) $\mathrm{SrTiO}_{3}$ при дальнейшем изготовлении мезаструктур (в случае применения ионного травления) может становиться проводящей.

Слой YВСО напылялся непосредственно на подложку при $830^{\circ} \mathrm{C}$ и давлении кислорода $0.5 \mathrm{mbar}$, последующий слой $\mathrm{Sr}_{2} \mathrm{IrO}_{4}$ наносился в атмосфере аргона при давлении $0.5 \mathrm{mbar}$ и температуре $700^{\circ} \mathrm{C}$. После охлаждения камеры до $500^{\circ} \mathrm{C}$ двухслойная гетероструктура выдерживалась в атмосфере кислорода в течение $30 \mathrm{~min}$. Далее наносился защитный слой золота. Все слои напылялись в одной камере и в одном технологическом цикле. На рис. 2 представлены $\Theta / 2 \Theta$ дифрактограммы для пленки $\mathrm{Sr}_{2} \mathrm{IrO}_{4} / \mathrm{NdGaO}_{3}$ и гетероструктуры $\mathrm{Au}(5 \mathrm{~nm}) / \mathrm{Sr}_{2} \mathrm{IrO}_{4}(10 \mathrm{~nm}) / \mathrm{YBCO}(60 \mathrm{~nm}) / \mathrm{NdGaO}_{3}$. Слой ҮВСО растет на $\mathrm{NdGaO}_{3}$ эпитаксиально с осью с перпендикулярной плоскости подложки, что подтверждается снятием дифрактограмм для разных отражений при наклоне образца и сканировании по азимутальному углу $\varphi$, аналогичным образом на $\mathrm{YBCO}$ растет пленка $\mathrm{Sr}_{2} \mathrm{IrO}_{4}$. Значение $c$-параметра $\mathrm{Sr}_{2} \mathrm{IrO}_{4}$ остается близким к табличным (рис. 2,b), температура перехода в сверхпроводящее состояние слоя ҮВСО также не изменяется относительно автономной пленки и составляет $91 \mathrm{~K}$.

Таким образом, была отработана технология эпитаксиального роста метастабильной фазы иридата $\mathrm{Sr}_{2} \mathrm{IrO}_{4}$ и двухслойных структур купратный сверхпроводник - иридат стронция в комбинированной

Письма в ЖТФ, 2017, том 43, вып. 12 

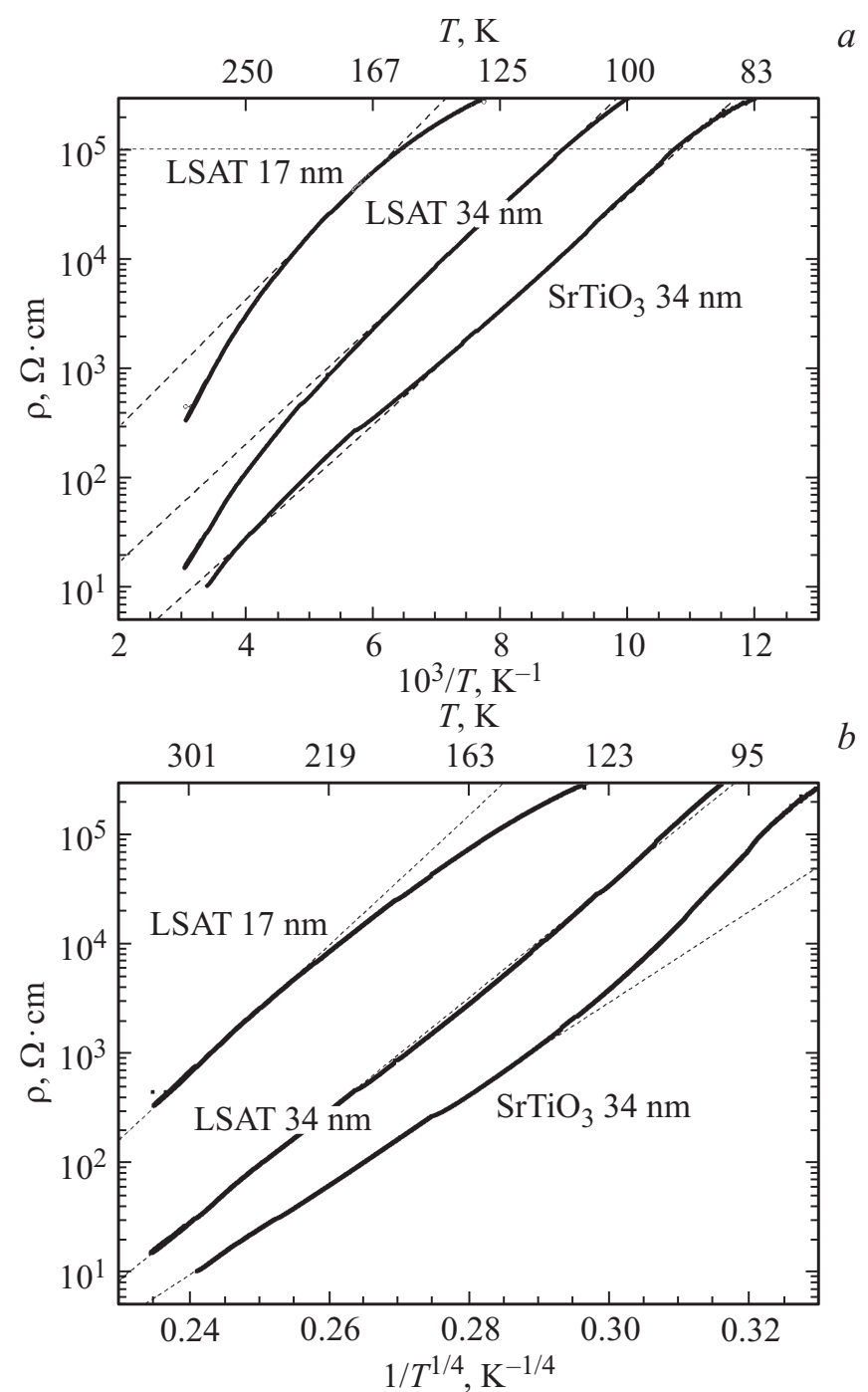

Рис. 3. Зависимость удельного сопротивления пленок $\mathrm{Sr}_{2} \mathrm{IrO}_{4}$, нанесенных на подложки LSAT (толщиной 17 и $34 \mathrm{~nm}$ ) и $\mathrm{SrTiO}_{3}$ (толщиной $34 \mathrm{~nm}$ ) в следующих координатах: $(a)-\rho(1000 / T), b-\rho\left(T^{-1 / 4}\right)$. 
аргон-кислородной атмосфере. Показано, что критическая температура купратного сверхпроводника не снижается при напылении поверх него пленки из иридата, также остается практически неизменным $c$ параметр иридата. Кривые сопротивления автономных пленок иридата от температуры показывают активационный тип проводимости, при высоких температурах добавляется механизм 3-мерной прыжковой проводимости. Обнаружение активационного типа проводимости даже для тонких пленок может свидетельствовать о малом количестве дефектов в рассматриваемых пленках.

Работа была поддержана проектом РФФИ 16-37-60069, 14-07-00258 и научной школой НШ-8168.2016.2. Авторы благодарны А.В. Шадрину, Т.А. Шайхулову, К.И. Константиняну, Л.В. Филиппенко, Т.А. Свиридовой, а также Stephan Schmid и Birgit Lemke за помощь в проведении эксперимента и полезные обсуждения.

\section{Список литературы}

[1] Kim B.J., Ohsumi H., Komesu T. et al. // Science. 2009. V. 323. P. 1329.

[2] Kim Y.K. et al. // Nat. Phys. 2016. V. 12. P. 37.

[3] Ge M., Qi T.F., Korneta O.B. et al. // Phys. Rev. B. 2011. V. 84. P. 100402(R).

[4] Hasan M.Z., Kane C.L. // Rev. Mod. Phys. 2010. V. 82. P. 3045.

[5] Mats Horsdal, Giniyat Khaliullin, Timo Hyart, Bernd Rosenow // Phys. Rev. B. 2016. V. 93. P. 220502(R).

[6] Feng Ye, Songxue Chi, Bryan C. Chakoumakos et al. // Phys. Rev. B. 2013. V. 87. P. $140406(\mathrm{R})$.

[7] Lupascu A. et al. // Phys. Rev. Lett. 2014. V. 112. P. 147201.

[8] Chengliang Lu, Andy Quindeau, Hakan Deniz et al. // Appl. Phys. Lett. 2014. V. 105. P. 082407.

[9] Nichols J., Terzic J., Bittle E.G. et al. // Appl. Phys. Lett. 2013. V. 102. P. 141908.

[10] Rayan Serrao C. et al. // Phys. Rev. B. 2013. V. 87. P. 085121.

[11] Овсянников Г.А., Шейерман А.Е., Шадрин А.В. и др. // Письма в ЖЭТФ. 2013. T. 97. B. 3. C. 165-169.

[12] Randall et al. // J. Am. Chem. Soc. 1957. V. 79. P. 266.

[13] Perovskite Materials - Synthesis, Characterisation, Properties, and Applications / Book ed. by Likun Pan and Guang Zhu. ISBN 978-953-51-2245-6. Publ.: February 3, 2016.

[14] Bebenin N.G. et al. // J. Phys.: Condens. Matter. 2005. V. 17. P. 5443.

3 Письма в ЖТФ, 2017, том 43, вып. 12 